\title{
Grupo de orientação postural a idosos com dor osteomuscular: estabelecendo relações entre teoria e prática*
}

\section{Orientation posture group to elderly with osteomuscular pain: making relations between theory and practice}

\author{
Maria Carolina Agnolon', Simone Silva e Santos², \\ Maria Helena Morgani de Almeida ${ }^{3}$
}

AGNOLON, M. C.; SANTOS, S. S.; ALMEIDA, M. H. M. Grupo de orientação postural a idosos com dor osteomuscular: estabelecendo relações entre teoria e prática. Rev. Ter. Ocup. Univ. São Paulo, v. 17, n. 2, p. 80-86, maio/ago., 2006.

\begin{abstract}
RESUMO: O envelhecimento populacional acompanha-se de alta prevalência de doenças e dores crônicas que reduzem a capacidade funcional dos idosos. A abordagem do terapeuta ocupacional a portadores de dor crônica deve voltar-se para a superação da dor, estimulação e adoção de habilidades de autocuidado. Conduzimos descrição e análise de uma intervenção em grupo com idosos portadores de dor osteomuscular enquanto possível estratégia para o desenvolvimento de habilidades de autocuidado nesta população. A intervenção grupal combinou entrevistas individuais com foco na presença de dor nas atividades cotidianas; oito sessões semanais, com duração de uma hora, durante as quais foram realizadas observações do desempenho funcional dos idosos, demonstrações, pelos próprios participantes e coordenadoras, de maneiras alternativas para o desempenho de atividades realizadas com dor e práticas de massagem para prevenção e alívio da dor. Foram adotados, como base para a análise desta experiência, os Modelos conceituais do Autocuidado e a Classificação Internacional de Funcionalidade, Incapacidade e Saúde. No resgate da experiência do grupo verificamos ressonância entre aspectos da prática e conceitos veiculados pelos modelos teóricos adotados. Constatamos que as informações compartilhadas no grupo, possibilitaram aos idosos identificar posturas, movimentos e arranjos ambientais geradores de dor e ainda, superar ou reduzir a dor no desempenho de algumas atividades, entretanto os participantes apontam dificuldades em realizar mudanças posturais e ambientais duradouras. Embora reconheçamos o grupo de orientação postural como uma estratégia para desenvolver habilidades de autocuidado e melhorar a capacidade funcional de idosos com dor osteomuscular, este deve ser complementada por outras formas de abordagem.
\end{abstract}

DESCRITORES: Idoso. Terapia ocupacional. Grupos etários. Postura. Dor. Envelhecimento da população.

\footnotetext{
*Monografia apresentada para conclusão de Curso de Graduação em Terapia Ocupacional da FMUSP.

${ }^{1}$ Terapeuta Ocupacional, residente multiprofissional em Saúde da Família pelo Ministério da Saúde/Casa de Saúde Santa Marcelina - SP

${ }^{2}$ Terapeuta Ocupacional, aprimoramento em Ortopedia e Traumatologia e especialização em Terapia da Mão pelo IOT-HCFMUSP.

${ }^{3}$ Professora Doutora do Departamento de Fisioterapia, Fonoaudiologia e Terapia Ocupacional da FMUSP, Doutora em Saúde Pública pela Faculdade de Saúde Pública da USP.

Endereço para correspondência: Maria Helena Morgani de Almeida, Departamento de Fisioterapia, Fonoaudiologia e Terapia Ocupacional da FMUSP. Rua Cipotânea, 51. CEP: 05360-000. São Paulo, SP. e-mail: hmorgani@usp.br
} 


\section{INTRODUÇÃO}

$\mathrm{N}$

este estudo buscamos descrever uma intervenção em grupo com idosos para redução de dor osteomuscular no desempenho de atividades cotidianas e analisar esta experiência enquanto estratégia para o desenvolvimento de habilidades de autocuidado nesta população.

Orem (1991) define Autocuidado como a prática de atividades que indivíduos iniciam e desempenham em seu próprio benefício, a fim de manter a vida, a saúde e o bem estar. O Autocuidado se constitui como importante mecanismo para a promoção da saúde, especialmente em idosos, uma vez que por meio dele é possível prevenir enfermidades e incapacidades (RESTREPO; PEREZ, 1994).

O envelhecimento populacional é acompanhado pelo aumento da susceptibilidade para enfermidades crônicas e incapacidades (LITVAK, 1990). Estudos sobre capacidade funcional, conduzidos no Brasil na década de 1990, encontraram altas prevalências de incapacidades para, pelo menos, uma atividade de vida diária, que variam de $28 \%$ a 61\% (LITVOC; DERNTL, 2002).

Segundo De Carlo (2004), a incapacidade funcional pode ser decorrente de processos dolorosos, que prejudicam a autonomia e a independência da pessoa idosa para as atividades diárias, diminuindo sua funcionalidade e consequientemente prejudicando sua qualidade de vida. Para Yeng (2001), o tratamento da dor requer intervenções em outros campos além do clínico, com o objetivo de melhora da qualidade de vida e não apenas diminuição do desconforto físico provocado pela dor. É nesse contexto que a terapia ocupacional atua.

Para a terapia ocupacional, o tratamento da dor crônica em idosos deve ser baseado na solução de problemas e atividades funcionais que os idosos possam aprender. Segundo Carpenter et al. (2001), o tratamento da dor crônica requer uma intervenção distinta da atenção baseada no modelo médico utilizada no tratamento da dor aguda, devese voltar para uma atenção mais compreensiva de superação da dor, estimulação e adoção de habilidades de autocuidado. Consideramos que o desenvolvimento de habilidades de autocuidado possa ser facilitado pela intervenção grupal. Hasselkus (1998) preconiza a abordagem em grupo para idosos e pacientes com problemas crônicos de saúde, pois o grupo possibilita a promoção da participação ativa de seus participantes no processo terapêutico.

Reconhecendo o grupo como facilitador da participação de seus membros e inferindo que o compartilhar da experiência da dor e de alternativas para sua redução ou superação pudesse intensificar-se numa situação grupal, com repercussões favoráveis na vida cotidiana dos participantes, conduzimos intervenção grupal com idosos portadores de dor osteomuscular. O objetivo deste estudo é descrever esta experiência e analisar seu potencial terapêutico, especialmente para o desenvolvimento de habilidades de autocuidado em idosos. Para essa análise, adotamos como principais referenciais teóricos, os modelos do Autocuidado e a Classificação Internacional de Funcionalidade, Incapacidade e Saúde.

\section{DESENVOLVIMENTO DO GRUPO DE ORIENTAÇÃO POSTURALAIDOSOS COMDOROSTEOMUSCULAR}

Desenvolvemos um grupo de orientação postural para idosos com dor osteomuscular crônica, como parte de estágio extracurricular de Terapia Ocupacional. Esta experiência foi desenvolvida no ano de 2003, num centro de reabilitação pertencente a um hospital escola da cidade de São Paulo. O objetivo deste grupo era promover a redução da dor referida pelos idosos no desempenho de suas atividades cotidianas. A metodologia empregada consistiu na aplicação, , de formulário sobre presença e grau de dor nas atividades cotidianas, por meio de entrevista individual; oito sessões grupais, de freqüência semanal com duração de uma hora, durante as quais eram realizadas, sistematicamente, observações do desempenho funcional dos idosos, demonstrações, pelos próprios participantes e coordenadoras, de maneiras alternativas para o desempenho de atividades realizadas com dor e práticas de massagem para prevenção e alívio da dor.

A entrevista individual inicial buscou identificar as demandas cotidianas relacionadas à dor, e ao término dos atendimentos, para avaliação das possíveis mudanças quanto às demandas inicialmente apresentadas. A freqüência de dor durante a realização de atividades foi o principal critério para a seleção das atividades propostas.

Identificamos três momentos distintos nas sessões. Num primeiro momento, um integrante do grupo realizava a atividade proposta, enquanto os outros observavam. Num segundo momento, o grupo descrevia como a mesma atividade poderia ser realizada de uma forma mais econômica, segura e confortável. Num terceiro momento, a atividade era realizada novamente, quando propúnhamos alterações na forma de realizar a atividade, com base nas modificações que já haviam sido apresentadas pelo grupo e na literatura que enfatiza a importância da conservação de energia, proteção articular, ergonomia e cinesiologia para a realização das atividades de vida diária. 


\section{RESULTADOS}

Participaram do grupo de orientação postural, oito indivíduos usuários do centro de reabilitação e encaminhados pelo serviço médico da instituição. Os idosos tinham entre 69 e 79 anos; seis eram mulheres e dois eram homens, seis eram aposentados e referiram como atividades profissionais predominantes em suas vidas, atividades que implicaram em esforço físico (doméstica, costureira, faxineiro, copeira e donas de casa), no grupo havia ainda um engenheiro agrônomo aposentado.

De maneira geral, os idosos não freqüentavam ambientes públicos, sendo seu lazer limitado a visitas a casas de familiares e idas à igreja. As atividades cotidianas destes idosos focalizavam-se em básicas e instrumentais de vida diária.

Quanto à dor, o tempo de queixa variou de três meses a 35 anos. Cinco idosos relataram dor em mais de uma região corporal, sendo que a localização de maior prevalência referiu-se a coluna lombar. Cinco participantes relataram dor constante, desses, dois disseram sofrer alterações na qualidade do sono em função da dor.

Quando questionados sobre o que faziam para alívio da dor, relataram o uso de doses extras de medicação e interrupção da atividade que provocava a dor.

A dor era mais freqüente na realização das seguintes atividades e práticas cotidianas: vestuário, higiene pessoal, adoção de determinadas posturas e transferências posturais, atividades de limpeza e de manutenção da casa. Estas atividades foram utilizadas no grupo, tornando-se alvo de orientação, com vistas à sua melhor realização na vida cotidiana.

De maneira geral, o grupo realizou as atividades propostas, embora tenha havido resistência em alguns momentos em função da dor ou do gênero. $O$ desempenho dos idosos em algumas atividades e orientações posturais para superação ou redução da dor, são apresentados, de forma sucinta, no Quadro 1.

Nas entrevistas conduzidas ao final do processo, os idosos referiram que, de maneira geral, a experiência vivenciada foi de grande importância e que as orientações os ajudaram a identificar no dia-a-dia as posturas, movimentos e arranjos ambientais geradores de dor. As orientações ajudaram ainda a superar ou reduzir a dor no desempenho de algumas atividades cotidianas, conforme apresentado no Quadro 2. Os idosos alegaram entretanto que é difícil incorporar novos hábitos ao cotidiano e realizar mudanças posturais e ambientais duradouras.

\section{DISCUSSÃO}

No processo de avaliação sobre a experiência do grupo de orientação postural em suas distintas etapas foi possível estabelecer algumas relações entre aspectos da prática e conceitos veiculados pelos Modelos teóricos do Autocuidado e Classificação Internacional de Funcionalidade, Incapacidade e Saúde.

A principio, para a realização de grupos futuros, consideramos a importância da re-elaboração do formulário adotado nas entrevistas individuais, de modo que este venha contemplar aspectos que, após o conhecimento dos modelos e outros referenciais teóricos, julgamos relevantes para nortear o desenvolvimento de um grupo que tenha como propósito desenvolver habilidades de autocuidado de idosos em processo de dor osteomuscular. O novo formulário deverá prever levantamento sistemático de características da dor e possibilitar sua avaliação como desencadeante de limitações de atividades e restrições de participação social. Esta avaliação deverá contribuir para prevenção destas condições, enquanto estágios previstos pela Classificação Internacional de Funcionalidade, Incapacidade e Saúde (CIF).

ACIF foi elaborada pela OMS (2003) e procura identificar os componentes que constituem a saúde e descrever o impacto das doenças nas condições de vida das pessoas. Os fatores de saúde e relacionados a ela são descritos na CIF com base no corpo, no indivíduo e na sociedade.

Uma crítica importante ao formulário de avaliação, a partir da CIF, refere-se à ausência de informações referentes ao ambiente. Este habitualmente interfere nas ações e no desempenho dos sujeitos em suas atividades cotidianas, se tornando muitas vezes uma barreira para a sua participação nestas atividades. Para esta classificação "a funcionalidade e a incapacidade de uma pessoa são concebidos como produto da interação dinâmica entre os estados de saúde (doenças, distúrbios, lesões, traumas, etc.) e os fatores contextuais (pessoais e ambientais)" (OMS, 2003, p.19).

Observamos os limites impostos pelo corpo durante a realização das atividades do grupo e buscamos valorizar as estratégias desenvolvidas pelos idosos para superar esses limites, formulando as orientações prestadas aos participantes, a partir das práticas adotadas e compartilhadas no grupo pelos mesmos. Segundo Chang (1980) e Orem (1991) fatores culturais, étnicos e sociais influenciam o autocuidado e devem ser levados em consideração pelo profissional de saúde, no momento de sua intervenção. Orem (1991) acrescenta que o cuidado que o homem é capaz de prestar a si próprio é aprendido ao longo do seu desenvolvimento e amadurecimento. 
Quadro 1 - Desempenho pelos idosos de algumas atividades para as quais relataram queixa de dor na avaliação inicial e orientações posturais prestadas para superação ou redução da dor.

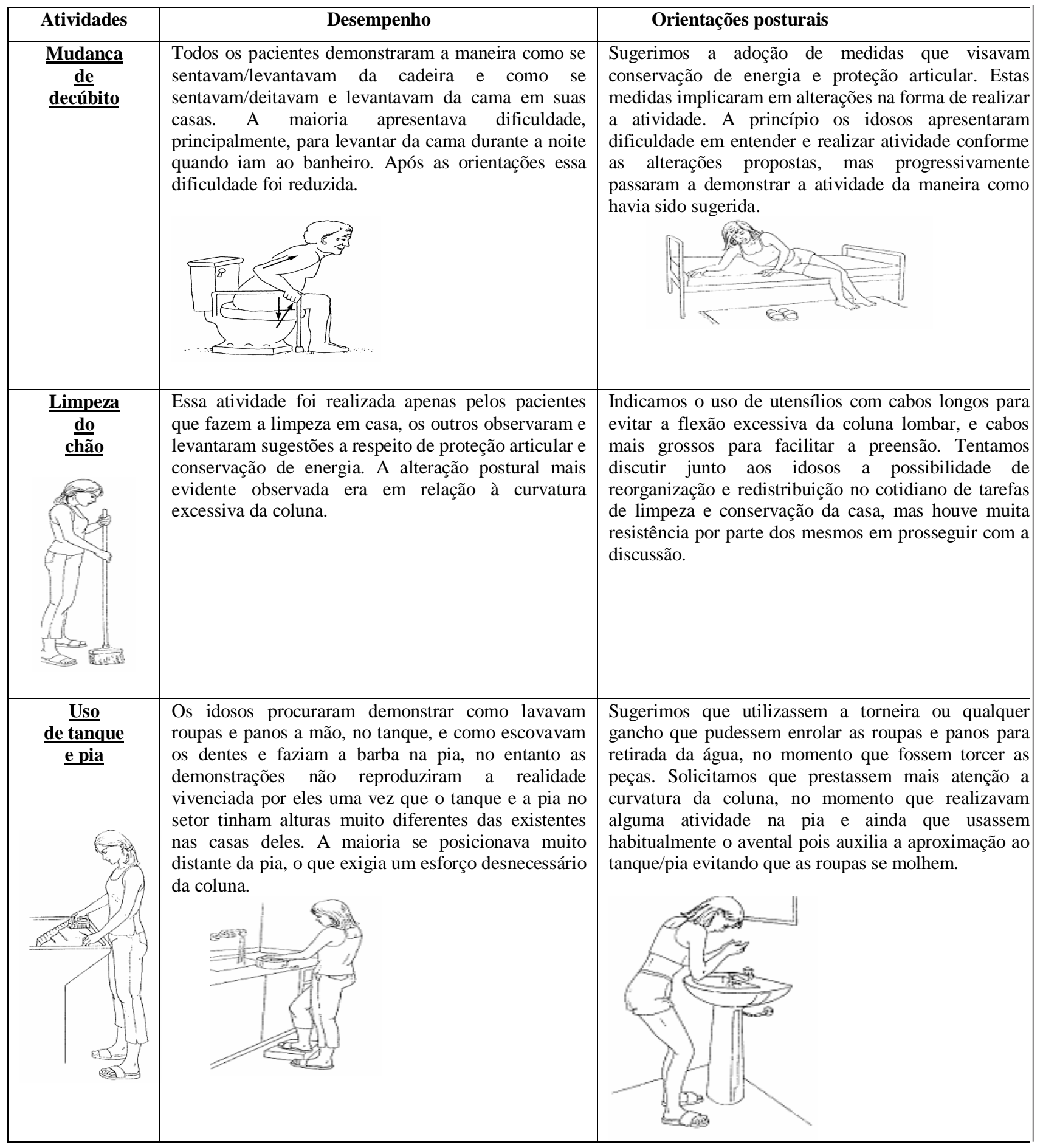


AGNOLON, M. C. et al. Grupo de orientação postural a idosos. Rev. Ter. Ocup. Univ. São Paulo, v. 17, n. 2, p. 80-86, maio/ago., 2006.

Quadro 2 - Número de idosos segundo presença e grau da dor no desempenho das atividades cotidianas na avaliação inicial e final.

\begin{tabular}{|c|c|c|c|c|}
\hline \multirow{3}{*}{ Atividades Realizadas } & \multicolumn{4}{|c|}{ Resultados da Avaliação } \\
\hline & \multirow[t]{2}{*}{ Inicial } & \multicolumn{3}{|c|}{ Final } \\
\hline & & Dor reduzida & Sem dor & Dor inalterada \\
\hline Lavar louças & 6 & 3 & 2 & 1 \\
\hline Vestir meia & 4 & 1 & 2 & $1 *$ \\
\hline Calçar sapato & 3 & 1 & 2 & 0 \\
\hline Lavar roupa (esfregar e torcer) & 4 & 3 & 0 & 1 \\
\hline Deitar e levantar-se - sanitário & 5 & 4 & 1 & 0 \\
\hline Deitar e levantar-se - cama & 4 & 3 & 1 & 0 \\
\hline Varrer e passar pano no chão & 6 & 4 & 1 & $1 *$ \\
\hline
\end{tabular}

* 1 pessoa não realizava a atividade por causa da dor

Algumas práticas compartilhadas pelos idosos no grupo constituíram-se de fato em práticas de autocuidado e envolveram mudanças na forma de realizar algumas atividades cotidianas e mudanças ambientais. Estas mudanças contribuíram para a redução da dor durante a realização das atividades. Destacamos que alguns idosos abandonavam determinadas atividades quando sua realização comprometia o bem estar. Algumas habilidades de autocuidado foram sugeridas pelos demais participantes e coordenadoras, com vistas à realização destas atividades com menor dificuldade e sua manutenção no universo ocupacional do idoso.

A teoria do Autocuidado elaborada por Orem (1991) articula conceitos tais como "necessidades de autocuidado", "competência e déficit para o autocuidado" e "demanda terapêutica de autocuidado". De acordo com Neufeld e Hobbs (1985) necessidades de autocuidado acompanham os indivíduos durante toda a sua vida, sendo que algumas são geradas e modificadas pelo processo de envelhecimento. Muitas vezes este processo é acompanhado por doenças, dores crônicas e incapacidades geradoras de necessidades de autocuidado específicas e de uma demanda terapêutica através da qual estas necessidades poderão ser satisfeitas. Esta demanda poderá incluir métodos e técnicas selecionados, tais como modificações comportamentais e tecnologia assistiva.

Com base nestes conceitos podemos afirmar que a dor pode comprometer a competência de idosos para autocuidado, gerando déficits e demanda terapêutica que compreenderão, dentre outras estratégias, modificações internas dirigidas ao comportamento e modificações externas relativas ao ambiente.

De acordo com Almeida (2003) a administração do próprio cotidiano pelo indivíduo configura-se como prática de autocuidado. Alguns estudos citados pela autora têm se ocupado em identificar práticas adotadas por idosos e/ou cuidadores para minimizar ou corrigir déficits nas atividades cotidianas. Mudanças comportamentais, ambientais e uso de dispositivos auxiliares incluem-se nessas práticas (NORBURN et al., 1995; ALMEIDA, 1997; GILLet al., 1999; GITLIN etal., 2002).

Reconhecemos que o significado, para os idosos, dos limites corporais e o alcance das estratégias empregadas sobre sua participação social mais ampla não foram sistematicamente abordados durante a intervenção grupal.

Identificamos com base numa reflexão crítica, alguns fatores que determinaram nosso pouco investimento na abordagem dos significados da dor e dos limites corporais e sua relação com a participação social dos idosos: 1) a dificuldade em administrar as complexas demandas do grupo, e que não se limitavam ao alívio da dor e a necessidade de orientação postural; 2) nosso conhecimento, até então restrito, acerca do envelhecimento enquanto fenômeno complexo e que abrange aspectos biológicos mas fundamentalmente psicológicos, sociais e ambientais; 3 ) a falta de apreensão da dor como elemento que compõe o paradigma ainda vigente do idoso como individuo doente, improdutivo e incapaz, ou seja, a dor como fenômeno ao qual podem ser atribuídos distintos significados, avaliados em contextos específicos; 4) a resistência ou dificuldade, dos participantes, em promover mudanças em seu cotidiano. 
Buscando um entendimento acerca dos possíveis significados da resistência ou dificuldade dos idosos participantes em realizar mudanças em seu cotidiano, podemos inferir que pode ser decorrente de fatores como o próprio processo de envelhecimento e doenças que freqüentemente o acompanham e que contribuem, em diferentes graus, para a produção de déficits para o autocuidado; apego a valores e costumes fortemente arraigados e que constituem a própria identidade do idoso e escassez de apoio familiar e social para a promoção destas mudanças.

Conforme os idosos referiram, os conhecimentos recém adquiridos embora essenciais para desenvolver habilidades para o autocuidado, não são na maioria das vezes, suficientes para promover mudanças, persistentes e duradouras, de comportamentos fortemente arraigados, requerendo estratégias combinadas para o alcance desta finalidade.

Consideramos que estas mudanças devem ser fruto de reflexão, e do exercício da autonomia destes idosos, ou seja, devem ser percebidas como algo significativo e valioso, a fim de que o idoso busque alternativas para desenvolvimento de novas e eficientes estratégias para o autocuidado. Como vimos anteriormente, comportamentos são repletos de significados a serem desvendados e compreendidos e muitas vezes compõe a identidade do idoso, sendo adotados inclusive como mecanismos para manter sua integridade. Assim, orientações quanto a mudanças comportamentais e ambientais devem ser conduzidas com respeito a valores e costumes do idoso e refletir sua condição de ser autônomo e participante do processo de atenção a sua saúde.

Freqüentemente o idoso requer o apoio de familiares e amigos para empreender mudanças, nestes casos, muitas vezes torna-se necessário ir além do grupo, identificando a rede de suporte social deste idoso e buscando ativá-la e alimentá-la continuamente.

Consideramos importante ainda que, em futuros grupos com idosos com dor crônica, se adotem estratégias que desloquem a questão da dor, deficiência e incapacidade de sua dimensão individual e de responsabilização pessoal, em direção a sua compreensão como fenômeno social. Esta compreensão permite que os idosos sejam fortalecidos em seu papel de cidadãos, atuando coletivamente para identificação e intervenção sobre fatores produtores de incapacidades, a fim de minimizar ou mesmo eliminar barreiras à sua inclusão social.

\section{CONCLUSÃO}

A atenção aos idosos com dor osteomuscular em contexto grupal configurou-se como estratégia importante para o desenvolvimento de habilidades de autocuidado, no entanto, após a análise desta experiência, percebemos que alguns aspectos que aumentariam a resolutividade desta proposta e sua potencialidade como promotora de mudanças comportamentais e ambientais duradouras não foram contemplados. Consideramos que reflexões sobre a limitação na participação social, a dor como co-determinante desta limitação ou mesmo a exclusão social como produtora de dor são aspectos a serem sistematicamente contemplados.

Reconhecemos, a partir deste estudo, a necessidade de se adotar formas complementares ao grupo de orientação postural para desenvolver habilidades de autocuidado de idosos com dor osteomuscular que incluam abordagens mais compreensivas que contemplem, portanto, aspectos da subjetividade dos envolvidos, e outras formas em conformidade com a demanda e características da clientela e com a formação do terapeuta ocupacional.

AGNOLON, M. C.; SANTOS, S. S.; ALMEIDA, M. H. M. Orientation posture group to elderly with osteomuscular pain: making relations between theory and practice. Rev. Ter. Ocup. Univ. São Paulo, Rev. Ter. Ocup. Univ. São Paulo, v. 17, n. 2, p. 80-86, maio/ago., 2006.

\begin{abstract}
Among the growing population of elderly there is a high incidence of illness and chronic pain that reduce functional capacity of the elderly. The Occupational Therapist work with chronic pain patients looks for developing strategies to over come the pain, stimulation and adoption of the self-care practices. It was realized the description and analyze of group intervention with elderly with osteomuscular pain as the possible benefits in the development of self-care abilities. The group aids interviews, group's session, observation and demonstrations by elderly and coordinators about alternatives manners to do activities of daily life. We adopt two models: SelfCare and International Classification of Functionality, Incapacity and Health as theorical elements. In the rescue of the group's experience in the distinct steps, it was possible to relate theory and practice, especially with elements of the Models adopted. We verify that the information shared in the group made possible to the elderly to identify posture, movements and environment's factors generator of pain and also, over come or reduce pain during daily activities, although the participants show the difficulties on doing changes that last for long time. We recognize the group as a strategy to improve self-care abilities and functional capacity of elderly with osteomuscular pain, however it needs to be completed with other kinds of intervention
\end{abstract}

KEY WORDS: Aged. Occupational therapy. Pain. Posture. Demographic aging. Age groups. 


\section{REFERÊNCIAS}

ALMEIDA, M. H. M. C.I.C.Ac: instrumento para classificação de idosos quanto à capacidade para o autocuidado. 1997. Dissertação (Mestrado Saúde Pública) - Faculdade de Saúde Pública, Universidade de São Paulo, São Paulo, 1997.

ALMEIDA, M. H. M. Validação do C.I.C.Ac: instrumento para classificação de idosos quanto à capacidade para o autocuidado. 2003. Tese (Doutorado Saúde Pública) - Faculdade de Saúde Pública, Universidade de São Paulo, São Paulo, 2003.

CARPENTER, L.; BAKER, G. A.; TYLDESLEY, B. The use of the Canadian Occupational Performance Measure as an outcome of a Pain Mangement Program. Can. J. Occup. Ther., v. 68, n. 1, p. 16-22, 2001.

CHANG, B. L. Facilitating self care: review of the literature and a conceptual model. ANS, v. 3, n. 1, p. 43-58, 1980.

DE CARLO, M. M. R. P. O tratamento não farmacológico da dor e a abordagem do terapeuta ocupacional. São Paulo, 2004. [Texto utilizado na disciplina Estágio Supervisionado VII: Práticas Hospitalares em Terapia Ocupacional do Hospital Universitário da Universidade de São Paulo].

GILL, T. M.; ROBISON, J. T.; WILLIANS, C. S.; TINETTI, M. E. Mismatches between the home environment and physical capabilities among community-living older persons. J. Am. Geriatr. Soc., v. 47, p. 88-92, 1999.

GITLIN, L. N.; WINTER, L.; DENNIS, M. P.; CORCORAN, M.; SCHINFELD, S.; HAUCK, W. W. Strategies used by families to simplify tasks for individuals with alzheimer's disease and related disorders: psycometric analysis of task management strategy Index (TMSI). Gerontologist, v. 42, p. 61-9, 2002.
HASSELKUS, B. R. Discapacidad funcional en ancianos. In: HOPKINS, H. L.; SMITH, H. D. Terapia ocupacional de Willard e Spackman. 8a ed. Madrid: Panamericana, 1998. p. 742-52.

LITVAK, J. El envejecimiento de la población: un desafio que va más allá del año 2000. Bol. Of. Sanit. Panam., v. 109, p. 2-5, 1990.

LITVOC, J.; DERNTL, A. M. Capacidade funcional do idoso: significado e aplicações. In: CIANCIARULLO, T. I.; GUALDA, D. M. R.; SILVA, G. T. R.; CUNHA, I. C. K. O. Saúde na família e na comunidade. São Paulo: Robe editorial, 2002. p. 268-319.

NEUFELD, A.; HOBBS, H. Self-care in a high-rise for seniors. Nurs. Outlook, v. 33, p. 298-301, 1985.

NORBURN, J. E. K. I.; BERNARD, S. L.; KONRAD, T. R.; WOOMERT, A.; DEFRIESE, G. H.; KALSBEEK, W. D.; et al. Self-care and assistance from others in coping with functional status limitations among a national sample of older adults. $J$. Gerontol. B Psychol. Sci. Soc. Sci., v. 50, p. 101-9, 1995.

OREM, M. N. Nursing: concepts of practice. $4 \mathrm{a}$ ed. St. Louis: Mosby Year Book, 1991.

ORGANIZAÇÃO MUNDIAL DE SAÚDE - OMS. Classificação internacional de funcionalidade, incapacidade e saúde. Tradução: Cássia Maria Buchalla. São Paulo: EDUSP, 2003.

RESTREPO, H. E.; PEREZ, E. A. Promoción de la salud de los ancianos. In: PEREZ, E. A.; GALINSK, D.; MARTINEZ, A. R. S.; AYÉNDEZ, M. S. La atención de los ancianos: un desafio para los años noventa. Washington, D.C.: OMS/OPS, 1994. (POS - Publicación Cientifica, 546).

YENG, L. T.; STUMP, P.; KAZIYAMA, H. H. S.; TEIXEIRA, M. J.; IMAMURA, M.; GREVE, J. M. D. Medicina física e reabilitação em doentes com dor crônica. Rev. Med. (São Paulo), v. 80, n. ed. esp. pt. 2, p. 245-255, 2001.

Recebido para publicação: Junho/2006

Aceito para publicação: Julho/2006 